\title{
Photoelectron spectra of solvated electrons in bulk water, methanol, and ethanol
}

\section{$\operatorname{AUTHOR}(\mathrm{S})$ :}

Horio, Takuya; Shen, Huan; Adachi, Shunsuke; Suzuki, Toshinori

\section{CITATION:}

Horio, Takuya ... [et al]. Photoelectron spectra of solvated electrons in bulk water, methanol, and ethanol. Chemical Physics Letters 2012, 535: 12-16

\section{ISSUE DATE:}

2012-05

URL:

http://hdl.handle.net/2433/155801

\section{RIGHT:}

(C) 2012 Elsevier B.V.; This is not the published version. Please cite only the published version.; この論文は出版社版でありません。引用の際に は出版社版をご確認ご利用ください。 


\section{Photoelectron spectra of solvated electrons in bulk water, methanol, and ethanol}

Takuya Horio ${ }^{\mathrm{a}-\mathrm{c}}$, Huan Shen ${ }^{\mathrm{a}-\mathrm{c}}$, Shunsuke Adachi ${ }^{\text {a }}$, Toshinori Suzuki ${ }^{\mathrm{a}-\mathrm{c}, *}$

a Department of Chemistry, Graduate School of Science, Kyoto University,

Kitashirakawa-Oiwakecho, Sakyo-Ku, Kyoto 606-8503, Japan

${ }^{\mathrm{b}}$ CREST, Japan Science and Technology Agency, Sanbancho, Chiyoda-ku, Tokyo 102-0075, Japan

${ }^{\mathrm{c}}$ RIKEN Advanced Science Institute, RIKEN, 2-1 Hirosawa, Wako, 351-0851, Japan

Photoelectron spectra of solvated electrons in bulk liquids were obtained at energy-resolution of 60 meV using a linear time-of-flight photoelectron spectrometer and a $100 \mathrm{kHz}$ ultraviolet femtosecond laser. Solvated electrons in $\mathrm{H}_{2} \mathrm{O}, \mathrm{D}_{2} \mathrm{O}$, methanol, and ethanol were generated by $226 \mathrm{~nm}$ excitation of the charge-transfer-to-solvent bands of $\mathrm{I}^{-}$in $0.1 \mathrm{M}$ NaI solutions, and the photoelectron spectra were measured using $260 \mathrm{~nm}$ pulses with a time delay of 2 ns. The electron binding energies and band shapes are discussed.

* Corresponding author: TEL 81-75-753-3971, FAX 81-75-753-3974.

E-mail address: suzuki@kuchem.kyoto-u.ac.jp (Toshinori Suzuki). 


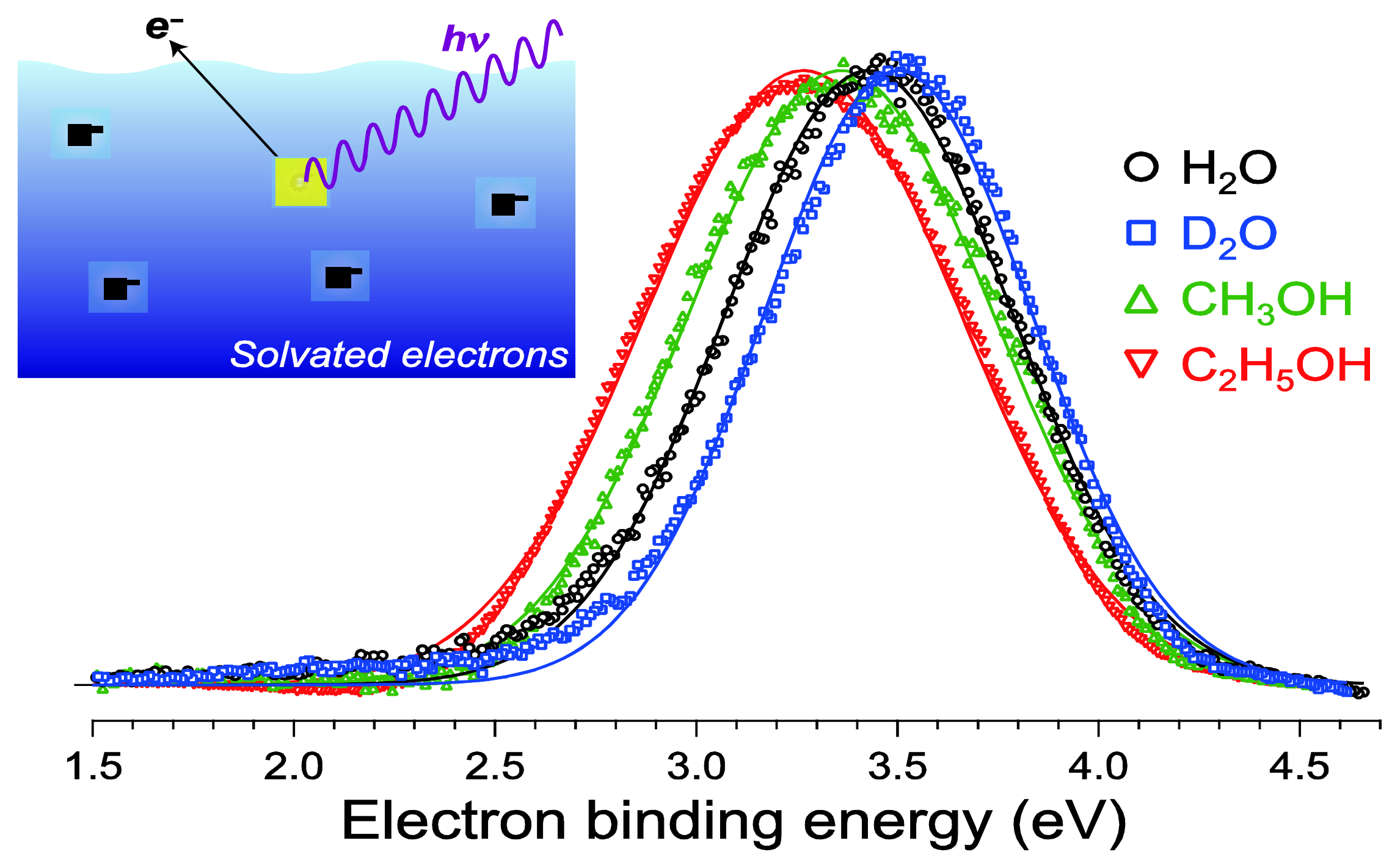




\section{Introduction}

Vertical electron binding energy (VBE) is one of the fundamental quantities of solvated electrons. VBEs of solvated electrons in bulk liquids, however, were not known, and they were estimated from the size-dependent VBE of cluster anions measured in the gas phase [1-4]. The validity of these estimates is debated, because some theoretical calculations suggest that excess electrons in these clusters are trapped at the cluster surface, and thus are not internalized [5-7]. Furthermore, since the VBEs of cluster anions are measured at ultralow temperatures, extrapolation of their VBEs to an infinitely large cluster size may give the VBEs of low-temperature solids rather than of room-temperature liquids.

In our previous studies, we measured the VBEs of solvated electrons in bulk water, methanol, and ethanol by time-resolved photoelectron spectroscopy (TRPES) of liquid beams using a hemispherical electron energy analyzer and a $1 \mathrm{kHz}$ ultraviolet femtosecond laser [8-11]. So far, four measurements have been reported for VBE of a hydrated electron by different groups, and the values range in 3.3 - $3.6 \mathrm{eV}$ [8,12-14]. Despite the remaining ambiguity, the experimentally measured VBEs of a hydrated electron have been used to investigate theoretical models, and a new delocalized model [15] was refuted because it predicts a much higher VBE than the measured value [15-17]. The bulk VBE values of a hydrated electron are in reasonable agreement with the prediction (3.3 eV [1]) based on VBEs of water cluster anions. The VBEs of solvated electrons in methanol and ethanol were reported to be $3.1 \mathrm{eV}$ only in our previous study [9]; this value is higher than the estimate $(2.6 \mathrm{eV})$ made from methanol cluster anions [3].

The discrepancy in the experimentally measured VBEs of a hydrated electron indicates experimental errors, which are possibly due to low signal-to-noise ratios, the short inelastic mean free path of an electron in liquid, the streaming potential of liquid $[10,18,19]$, and inelastic scattering of electrons in the gas phase. In order to achieve higher precision and reliability for TRPES of liquid, we have constructed a high-resolution time-of-flight (TOF) spectrometer coupled with a $100 \mathrm{kHz}$ deep ultraviolet femtosecond laser. In this letter, we report high-precision measurements of photoelectron spectra of solvated electrons in four polar protic solvents, namely $\mathrm{H}_{2} \mathrm{O}, \mathrm{D}_{2} \mathrm{O}$, methanol, and ethanol.

\section{Experimental Section}

Pump (226 nm) and probe $(260 \mathrm{~nm})$ pulses are generated from the output $(520 \mathrm{~nm})$ of a noncollinear optical parametric amplifier pumped by a $100 \mathrm{kHz}$ Ti:sapphire laser (output power: 7.5 W; pulse width: $70-80 \mathrm{fs}$ ) [20]. The cross-correlation time of the pump and probe pulses is 60 fs. The photoelectron kinetic energies in the photodetachment of solvated electrons at $260 \mathrm{~nm}$ are less than 3 eV, which gives a sufficiently high bulk-sensitivity [10]. Our new TOF analyzer (flight length: $1 \mathrm{~m}$ ) 
can be operated in a linear TOF or magnetic bottle TOF modes, and both provide symmetric Gaussian apparatus functions with the full width at half maximum of $20-30 \mathrm{meV}$ for gas samples. Although the magnetic bottle TOF mode [21,22] achieves a much higher count rate than the field-free linear TOF mode, we used a linear TOF mode in this study to minimize the observation of the electrons inelastically scattered by the evaporated gas around the liquid beam.

The vacuum system consists of a photoionization chamber, a liquid-beam dump chamber, and a TOF analyzer. The TOF analyzer has a graphite-coated entrance skimmer (diameter: $0.5 \mathrm{~mm}$ ) whose aperture is located at $2 \mathrm{~mm}$ from the ionization point. When a liquid beam is injected into the photoionization chamber, the chamber and TOF analyzer pressures are $(0.5-1.5) \times 10^{-3}$ and $<2 \times 10^{-6}$ Torr, respectively. The photoionization chamber and TOF analyzer are magnetically shielded by a 1-mm-thick permalloy inner layer and three layers of 1-mm-thick permalloy tubes, respectively. A 40-mm-diameter microchannel plate detector is placed at the end of the flight tube. The detector surface is biased at $+300 \mathrm{~V}$ to attract incident electrons, ensuring that the detection sensitivity is independent of the electron kinetic energy. The detector signal is amplified by a preamplifier and averaged using a multichannel scaler with a 2 ns bin. The detection system has an overall response time of less than 1 ns. The time origin $(t=0)$ of TOF measurements is determined with the accuracy better than 2 ns by scattered laser light.

The liquid beam is generated by discharging a solution at a flow rate of $0.5 \mathrm{~mL} / \mathrm{min}$ from a fused-silica capillary (inner diameter: $25 \mu \mathrm{m}$; length: $7 \mathrm{~mm}$ ) and the liquid beam is captured at a liquid- $\mathrm{N}_{2}$-cooled trap in the dump chamber. The outer surface of the capillary is coated with graphite to prevent surface charging. The laser beam diameters of the pump and probe beams are $0.2-0.3 \mathrm{~mm}$ at the illumination point, which is located $1 \mathrm{~mm}$ downstream from the nozzle. The average number of electrons detected per laser shot is $\sim 0.05$ for $0.1 \mathrm{M}$ aqueous NaI solution at the pump and probe pulse energies of 45 and $150 \mathrm{~nJ} /$ pulse, respectively. The analyzer is calibrated in the presence of a liquid beam using the photoelectron signals of one-color $(1+1)$ ionization of NO at $226 \mathrm{~nm}$ and two-color $(1+1$ ') ionization of NO using both 226 and $260 \mathrm{~nm}$ pulses. When observing these NO signals, the liquid beam is shifted $0.2 \mathrm{~mm}$ from the ionization point: the laser beam position was not moved. Since this procedure underestimates the streaming potential by $10 \%$, the true streaming potentials are obtained by multiplying the measured streaming potentials by a factor of 1.1.

In order to minimize the influence of the streaming potential on measurements of VBE, we employ a new experimental protocol in this study. We passivate fused-silica capillaries in air with sample solutions for more than 24 hours prior to TRPES experiments; we continuously record a streaming current with a picoammeter and select a well-passivated capillary for TRPES. The selected capillary is installed into the chamber, and VBEs of all solutions are measured using the same 
capillary nozzle and laser beam conditions. The streaming potential is measured before and after the VBE measurement of each sample solution to confirm stable experimental conditions during the measurement. The streaming potential is measured using $(1+1$ ') ionization of NO introduced from a gas nozzle. Figure 1 compares the electron TOF spectra obtained in the presence of different liquid beams. The different arrival times seen in the figure unambiguously demonstrate the generation of streaming potentials. Since the photoelectron kinetic energy distribution produced by $(1+1$ ') ionization of NO is inherently narrow, the observed band shape is entirely due to the apparatus function predominantly determined by the streaming potential. Figure 1 indicates our high-resolution TOF spectrometer has a Gaussian-shaped apparatus function, even in the presence of a liquid beam. The energy resolution degrades to $\sim 60 \mathrm{meV}$ for liquids from 20 - $30 \mathrm{meV}$ for gases owing to the streaming potentials.

\section{Results and discussion}

\subsection{Hydrated electron}

Previous studies have established that photoexcitation of the charge-transfer-to-solvent band of $\mathrm{I}^{-}$in water produces hydrated electrons well within 2 ns [11,23-26]. Figure 2 shows the electron TOF spectra observed for aqueous $0.1 \mathrm{M} \mathrm{NaI}$ solution in $\mathrm{H}_{2} \mathrm{O}$ for a pump-probe delay time of $2 \mathrm{~ns}$. The $2 \mathrm{~ns}$ is the longest time delay in TRPES of a hydrated electron reported so far, and it ensures equilibration of hydrated electrons prior to photodetachment. The decaying signals at $t<0.7 \mu$ s are caused by background photoemission in the flight tube illuminated by scattered laser light. The true electron signal from the liquid starts at ca. $0.8 \mu \mathrm{s}$. One-color background signals measured individually for the pump and probe laser pulses (red: $226 \mathrm{~nm}$; blue: $260 \mathrm{~nm}$ ) are subtracted from the observed signal (black) to obtain a two-color signal (green). Each signal and background spectrum was averaged over $3 \times 10^{7}$ laser shots and the results represent the averages of four sets of measurements.

The two-color TOF spectrum obtained was then transformed into a photoelectron spectrum (black in Figure 3). The abscissa in Figure 3 is the electron binding energy, which is given by the difference between the probe photon energy and the electron kinetic energy. The energy region of 0 $1.5 \mathrm{eV}$ is not shown, since no signal was observed in this region. Figure 3 also shows a similar result for a hydrated electron in $\mathrm{D}_{2} \mathrm{O}$ (blue). Both spectra have been calibrated against the streaming potentials $(-10-30 \mathrm{mV})$ of these liquid beams: the streaming potentials are rather small because we selected a $\mathrm{NaI}$ concentration at which the streaming potential is minimized.

Figure 3 clearly indicates that hydrated electrons in $\mathrm{D}_{2} \mathrm{O}$ and $\mathrm{H}_{2} \mathrm{O}$ have very similar VBEs, in agreement with our previous report [8]. In the present study, however, the VBE in $\mathrm{D}_{2} \mathrm{O}$ appears to 
be slightly larger than that in $\mathrm{H}_{2} \mathrm{O}$. The relative magnitudes of the VBEs determined are more reliable in this study than in our previous report [8], because the signal to noise ratio is considerably higher and measurements of two solutions were performed under the same experimental conditions, such as the same capillary nozzle and laser beam alignment. Both spectra in Figure 3 are very similar to Gaussian functions; the solid lines in the figure are best-fit Gaussian functions. The VBEs of hydrated electrons are evaluated as follows. The expectation values, $<\mathrm{E}>$, calculated from the observed distributions are $3.42 \pm 0.06$ and $3.47 \pm 0.05 \mathrm{eV}$ for $\mathrm{H}_{2} \mathrm{O}$ and $\mathrm{D}_{2} \mathrm{O}$, respectively. The band centers of Gaussian functions are $3.44 \pm 0.03$ and $3.49 \pm 0.03 \mathrm{eV}$ for $\mathrm{H}_{2} \mathrm{O}$ and $\mathrm{D}_{2} \mathrm{O}$, respectively. These results are summarized in Table 1. The spectra have full widths at half maxima of $0.84 \pm 0.05\left(\mathrm{H}_{2} \mathrm{O}\right)$ and $0.78 \pm 0.06 \mathrm{eV}\left(\mathrm{D}_{2} \mathrm{O}\right)$, which are in excellent agreement with our previous report, $0.86 \pm 0.06\left(\mathrm{H}_{2} \mathrm{O}\right)$ and $0.76 \pm 0.09 \mathrm{eV}\left(\mathrm{D}_{2} \mathrm{O}\right)$ [11]. The width determined for $\mathrm{H}_{2} \mathrm{O}(0.84 \mathrm{eV})$ in this study is smaller than the value, $1.1 \mathrm{eV}$, reported by Lubcke et al.[13].

\subsection{Solvated electrons in methanol and ethanol}

The charge-transfer-to-solvent reactions in methanol and ethanol are similar to that in water: photoexcitation creates solvated electrons well within 2 ns. Figure 4 shows the electron TOF spectra observed for $0.1 \mathrm{M} \mathrm{NaI}$ solution in methanol for a pump-probe time delay of $2 \mathrm{~ns}$. Each spectrum was obtained by averaging over $1.8 \times 10^{7}$ laser shots. One-color background signals were removed from the observed signal to obtain the true two-color signal, and three data sets were averaged. Figure 5 compares the photoelectron spectra of solvated electrons in methanol and ethanol with those in $\mathrm{H}_{2} \mathrm{O}$ and $\mathrm{D}_{2} \mathrm{O}$ (also shown in Figure 3). The band shapes observed for methanol and ethanol are also very similar to Gaussian functions. Close examination of Figure 4 reveals that one-color background signal is over-subtracted from the total signal and makes the two-color signal at around $1.0 \mu$ s slightly negative; this, however, influences the transformed distribution only slightly at around $2.0-2.5 \mathrm{eV}$. The VBEs of solvated electrons are determined, from the expectation values $<\mathrm{E}>$, to be $3.38 \pm 0.04$ and $3.30 \pm 0.03 \mathrm{eV}$ for methanol and ethanol, respectively. The central energies of the Gaussian functions are determined by least-squares fitting to be $3.36 \pm 0.02$ and $3.28 \pm 0.02 \mathrm{eV}$ for methanol and ethanol, respectively. The results of the present study confirm a finding of our previous report [9], namely that the solvated electrons in methanol and ethanol have larger VBEs than that estimate (2.6 eV [3]) from the VBEs of methanol cluster anions. The spectra have full widths at half maxima of $0.86 \pm 0.03$ (methanol) and $0.96 \pm 0.06 \mathrm{eV}$ (ethanol). $0.1 \mathrm{M} \mathrm{NaI}$ solution in methanol and ethanol had streaming potentials of +110 and $+120 \mathrm{mV}$, respectively: these potentials are relatively large, because we did not select the $\mathrm{NaI}$ concentration that minimizes the streaming potentials of methanol and ethanol. 


\subsection{Temperature dependence}

Previous studies have shown that photoabsorption spectra of solvated electrons in bulk solutions exhibit temperature dependence; the shifts of the absorption band maxima, $\mathrm{d} E / \mathrm{d} T$, are -2.4 and $-2.5 \mathrm{meV} / \mathrm{K}$ for a hydrated electron in $\mathrm{H}_{2} \mathrm{O}$ and $\mathrm{D}_{2} \mathrm{O}$ [27]. Similar temperature dependence may be expected for photoelectron spectra of solvated electrons. Thus, we heated the discharge nozzle from 293 to $333 \mathrm{~K}$ and examined the spectra of a hydrated electron in $\mathrm{H}_{2} \mathrm{O}$ and a solvated electron in methanol. No noticeable changes were observed in either case; an example is shown in Figure 6. The results suggest that the temperature dependences of the photoelectron band maxima, $d E / d T$, are not larger than $100 / 40=2.5 \mathrm{meV} / \mathrm{K}$ for a hydrated electron in $\mathrm{H}_{2} \mathrm{O}$ and a solvated electron in methanol. In future studies, we are planning to investigate the temperature dependence for a wider range.

\subsection{Symmetry of photoelectron bands}

The photoelectron spectra of solvated electrons in four different solvents, shown in Figure 5, are shifted and overlaid in Figure 7. The figure clearly shows symmetric band shapes of solvated electrons. It is noted that photoabsorption spectra of solvated electrons in bulk solutions and photoelectron spectra of water cluster anions exhibit asymmetric band shapes. Coe et al. have approximated the asymmetric photoelectron band shapes of water cluster anions (isomer I) using Gaussian and Lorentzian functions [28,29]; the Gaussian part is commonly expressed for $\mathrm{H}_{2} \mathrm{O}$ and $\mathrm{D}_{2} \mathrm{O}$ clusters anions (cluster size: $n=11-69$ ) by

$$
\left.I(E)=A \exp \left[-\left(E-E_{\text {peak }}\right)^{2} / 2 \sigma_{\mathrm{G}}{ }^{2}\right]\right]
$$

and

$\sigma_{\mathrm{G}}=-1.02 n^{-1 / 3}+0.548(\mathrm{eV})$.

These functions predict that the full width at half maximum of a photoelectron spectrum of a hydrated electron in bulk water is $1.29 \mathrm{eV}$. The widths observed in this study are smaller than this value. This comparison, however, is not totally adequate, because it is not established that isomer I is in internal (cavity) state [30]. As discussed by Coe et al. [31] and Ma et al. [4], the asymmetric band shapes of water cluster anions in the size range of $n=2-14$ are attributed in part to vibrational excitation of $\mathrm{OH}$ (or OD) stretch upon photodetachment; the photoelectron spectra of solvated electrons in bulk solutions exhibit no clear signature of the O-H (O-D) stretch band.

The ratio of the bandwidths observed for hydrated electron in $\mathrm{H}_{2} \mathrm{O}$ and $\mathrm{D}_{2} \mathrm{O}$ is $\sim 1$.1. Since the Franck-Condon region for photodetachment of a solvated electron is expected to be repulsive for intermolecular (solvation) coordinates, the Franck-Condon envelope reflects the ground-state 
wavefunction prior to photodetachment. The narrower spectrum for a hydrated electron in $\mathrm{D}_{2} \mathrm{O}$ than in $\mathrm{H}_{2} \mathrm{O}$ suggests a more confined nuclear wavefunction in the former owing to a smaller zero point energy and stronger hydrogen bonding. Although the freezing point of $\mathrm{D}_{2} \mathrm{O}$ is higher than $\mathrm{H}_{2} \mathrm{O}$, the narrower spectral width is not to be ascribed to the fact that aqueous $\mathrm{NaI}$ solution in $\mathrm{D}_{2} \mathrm{O}$ is slightly closer to its freezing point than aqueous $\mathrm{NaI}$ solution in $\mathrm{H}_{2} \mathrm{O}$, based on the small temperature dependence seen in Figure 6.

The difference of VBE between $\mathrm{H}_{2} \mathrm{O}$ and $\mathrm{D}_{2} \mathrm{O}$ has two possible sources. One is that a radius of gyration of an electron is slightly different between the two solvents (electronic effect) owing to stronger hydrogen-bonding network in $\mathrm{D}_{2} \mathrm{O}$. The other is that the vibrational frequencies of water molecules in the first hydration shell changes upon photodetachment, and that the differences of vibrational frequencies with and without an excess electron differ between the two solvents (vibrational effect). The relative contributions of these two factors are not clear at this point.

\section{Acknowledgment}

We would like to thank T. Mizuno and K. Sekiguchi for their contribution in constructing the new photoelectron spectrometer and N. Kurahashi for experimental assistance. 


\section{Table 1.}

Vertical binding energies $(\mathrm{eV})$ of solvated electrons. ${ }^{\text {a }}$

\begin{tabular}{|c|c|c|c|c|c|c|c|}
\hline \multirow{2}{*}{ Solvent } & \multicolumn{2}{|c|}{ Present study } & \multicolumn{5}{|c|}{ Ref. } \\
\hline & $<E>^{b}$ & Gauss fit & [8] & [9] & [12] & [13] & [14] \\
\hline $\mathrm{H}_{2} \mathrm{O}$ & $3.42(6)$ & $3.44(3)$ & 3.27 & - & $1.6 / 3.3$ & 3.4 & 3.6 \\
\hline $\mathrm{D}_{2} \mathrm{O}$ & $3.47(5)$ & $3.49(3)$ & 3.20 & - & - & - & - \\
\hline Methanol & $3.38(4)$ & $3.36(2)$ & - & 3.1 & - & - & - \\
\hline Ethanol & $3.30(3)$ & $3.28(2)$ & - & 3.1 & - & - & - \\
\hline
\end{tabular}

${ }^{a}$ Numbers in parentheses are $1 \sigma$ of the last digit.

${ }^{\mathrm{b}}$ Expectation values calculated from the observed photoelectron spectra.

\section{References}

[1] J.V. Coe, G.H. Lee, J.G. Eaton, S.T. Arnold, H.W. Sarkas, K.H. Bowen, C. Ludewigt, H. Haberland, D.R. Worsnop, J. Chem. Phys. 92 (1990) 3980.

[2] J.R.R. Verlet, A.E. Bragg, A. Kammrath, O. Cheshnovsky, D.M. Neumark, Science 307 (2005) 93.

[3] A. Kammrath, J.R.R. Verlet, G.B. Griffin, D.M. Neumark, J. Chem. Phys. 125 (2006) 171102.

[4] L. Ma, K. Majer, F. Chirot, B. von Issendorff, J. Chem. Phys. 131 (2009) 144303.

[5] L. Turi, W. S. Sheu, P. J. Rossky, Science 309 (2005) 914.

[6] O. Marsalek, F. Uhlig, T. Frigato, B. Schmidt, P. Jungwirth, Phys. Rev. Lett. 105 (2010) 043002.

[7] L. Mones, P. J. Rossky, L. Turi, J. Chem. Phys. 135 (2011) 084501.

[8] Y. Tang, H. Shen, K. Sekiguchi, N. Kurahashi, T. Mizuno, Y.-I. Suzuki, T. Suzuki, Phys. Chem. Chem. Phys. 12 (2010) 3653.

[9] H. Shen, N. Kurahashi, T. Horio, K. Sekiguchi, T. Suzuki, Chem. Lett. 39 (2010) 668.

[10] Y. Tang, Y.-I. Suzuki, H. Shen, K. Sekiguchi, N. Kurahashi, K. Nishizawa, P. Zuo, T. Suzuki, Chem. Phys. Lett. 494 (2010) 111.

[11] Y.-I. Suzuki, H. Shen, Y. Tang, N. Kurahashi, K. Sekiguchi, T. Mizuno, T. Suzuki, Chem. Sci. 2 (2011) 1094.

[12] K.R. Siefermann, Y.X. Liu, E. Lugovoy, O. Link, M. Faubel, U. Buck, B. Winter, B. Abel, Nat. Chem. 2 (2010) 274.

[13] A. Lubcke, F. Buchner, N. Heine, I.V. Hertel, T. Schultz, Phys. Chem. Chem. Phys. 12 (2010) 14629. 
[14] A.T. Shreve, T.A. Yen, D.M. Neumark, Chem. Phys. Lett. 493 (2010) 216.

[15] R.E. Larsen, W.J. Glover, B.J. Schwartz, Science 329 (2010) 65.

[16] L.D. Jacobson, J.M. Herbert, Science 331 (2011).

[17] L. Turi, A. Madarasz, Science 331 (2011) 1387.

[18] M. Faubel, B. Steiner, J.P. Toennies, J. Chem. Phys. 106 (1997) 9013.

[19] B. Winter, M. Faubel, Chem. Rev. 106 (2006) 1176.

[20] H. Shen, S. Adachi, T. Horio, T. Suzuki, Opt. Exp. 19 (2011) 22637.

[21] P. Kruit, F.H. Read, J. Phys. E. 16 (1983) 313.

[22] F. Buchner, A. Lubcke, N. Heine, T. Schultz, Rev. Sci. Instrum. 81 (2010) 113107.

[23] J.A. Kloepfer, V.H. Vilchiz, V.A. Lenchenkov, A.C. Germaine, S.E. Bradforth, J. Chem. Phys. 113 (2000) 6288.

[24] J.A. Kloepfer, V.H. Vilchiz, V.A. Lenchenkov, X.Y. Chen, S.E. Bradforth, J. Chem. Phys. 117 (2002) 766.

[25] V.H. Vilchiz, J.A. Kloepfer, A.C. Germaine, V.A. Lenchenkov, S.E. Bradforth, J. Phys. Chem. A 105 (2001) 1711.

[26] H. Iglev, A. Trifonov, A. Thaller, I. Buchvarov, T. Fiebig, A. Laubereau, Chem. Phys. Lett. 403 (2005) 198.

[27] F.Y. Jou, G.R. Freeman, J. Phys. Chem. 83 (1979) 2383.

[28] J.V. Coe, Int. Rev. Phys. Chem. 20 (2001) 33.

[29] J.V. Coe, S.M. Williams, K.H. Bowen, Int. Rev. Phys. Chem. 27 (2008) 27.

[30] L.D. Jacobson, J.M. Herbert, J. Am. Chem. Soc. 133 (2011) 19889.

[31] J.V. Coe, S.T. Arnold, J.G. Eaton, G.H. Lee, K.H. Bowen, J. Chem. Phys. 125 (2006) 014315. 


\section{Figure captions}

Figure 1. Electron time-of-flight spectra observed for $(1+1$ ') resonance two-photon ionization of NO in the presence of liquid beams of $0.1 \mathrm{M} \mathrm{NaI}$ solution in $\mathrm{H}_{2} \mathrm{O}$ (black), $\mathrm{D}_{2} \mathrm{O}$ (blue), methanol (green), and ethanol (red). The intensity is in arbitrary units. The solid lines show Gaussian functions obtained by a least-squares fit. The widths of TOF signal correspond to the energy width of $\sim 60 \mathrm{meV}$.

Figure 2. Electron time-of-flight spectra observed for $0.1 \mathrm{M}$ aqueous $\mathrm{NaI}$ solution in $\mathrm{H}_{2} \mathrm{O}$ at the pump-probe delay time of 2 ns. The intensity is in arbitrary units. Two-color signals (green) were obtained by subtraction of one-color spectra (red: $226 \mathrm{~nm}$ and blue: $260 \mathrm{~nm}$ ) from the spectrum measured with both 226 and 260-nm pulses (black).

Figure 3. Photoelectron spectra of hydrated electrons in $\mathrm{H}_{2} \mathrm{O}$ (black) and $\mathrm{D}_{2} \mathrm{O}$ (blue). The intensity is in arbitrary units. The solid lines show Gaussian functions obtained by a least-squares fit.

Figure 4. Electron time-of-flight spectra observed for $0.1 \mathrm{M} \mathrm{NaI}$ solution in $\mathrm{CH}_{3} \mathrm{OH}$ at the pump-probe delay time of 2 ns. The intensity is in arbitrary units. Two-color signals (green) were obtained by subtraction of one-color spectra (red: $226 \mathrm{~nm}$ and blue: $260 \mathrm{~nm}$ ) from the spectrum measured with both 226 and 260-nm pulses (black).

Figure 5. Photoelectron spectra of solvated electrons in four polar protic solvents. The intensity is in arbitrary units. The solid lines are Gaussian functions obtained by a least-squares fit.

Figure 6. Photoelectron spectra of hydrated electrons in $\mathrm{H}_{2} \mathrm{O}$ measured at different nozzle temperatures. The intensity is in arbitrary units. The abscissa corresponds to the relative energy with respect to the peak position of the observed photoelectron spectrum at the nozzle temperature of $293 \mathrm{~K}$ (black). The spectra measured at three temperatures are identical within the experimental uncertainty.

Figure 7. Photoelectron spectra of solvated electrons in four polar protic solvents. The intensity is in arbitrary units. The same spectra shown in Figure 5 are also shifted and overlaid. The solid lines are Gaussian functions obtained by a least-squares fit. Lower panel shows the residues of fits. 


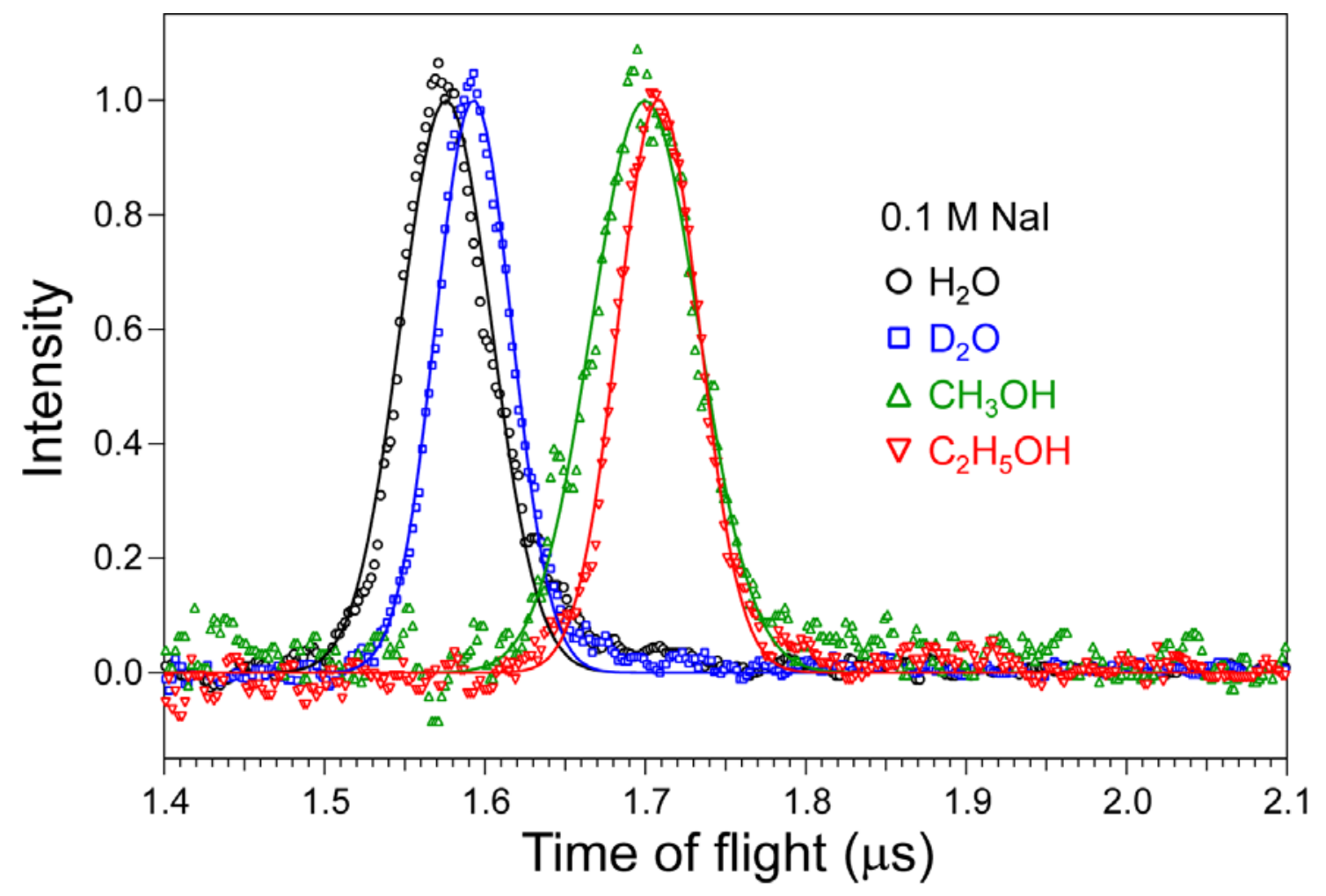

Figure 1. Horio et al

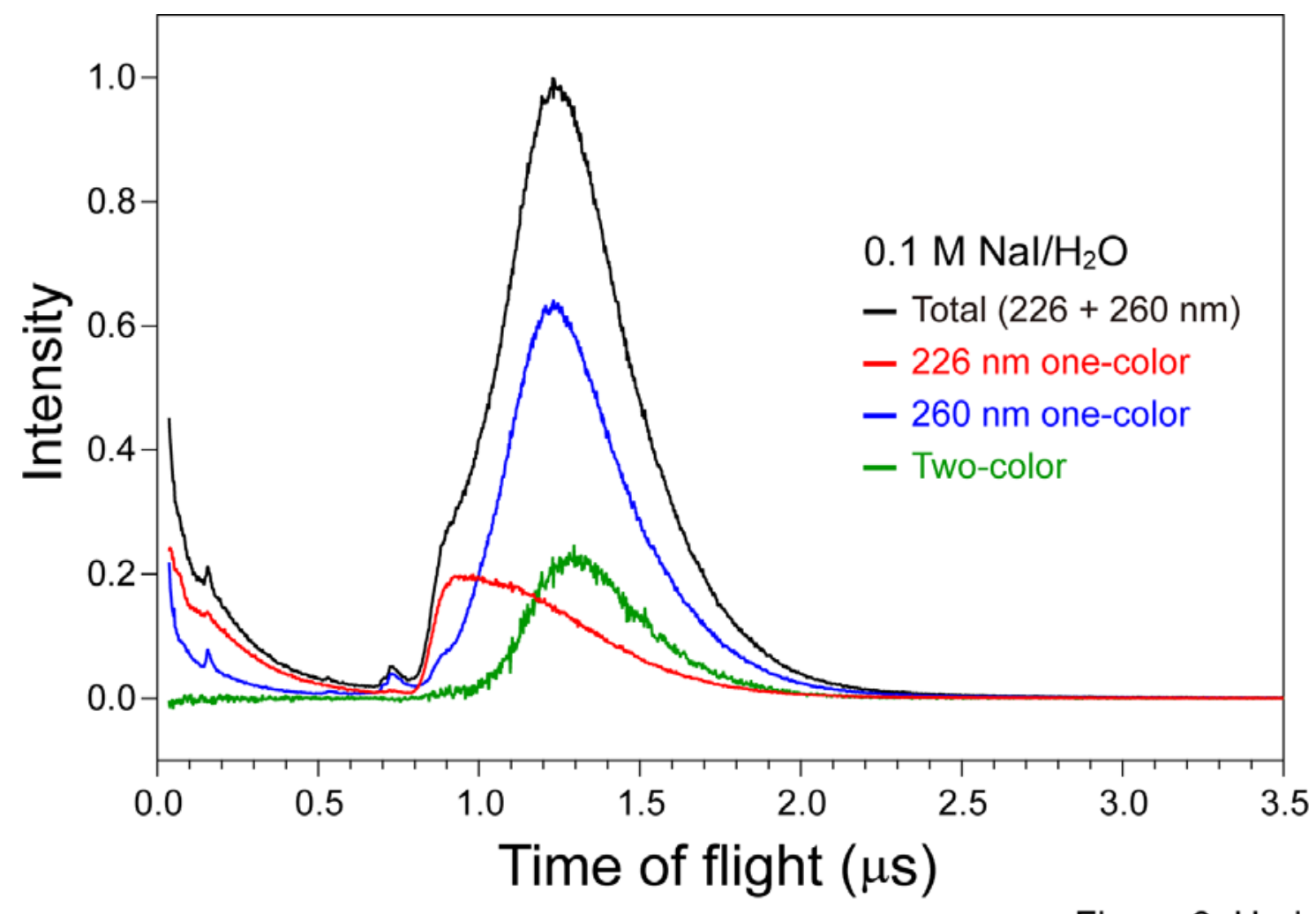

Figure 2. Horio et al 


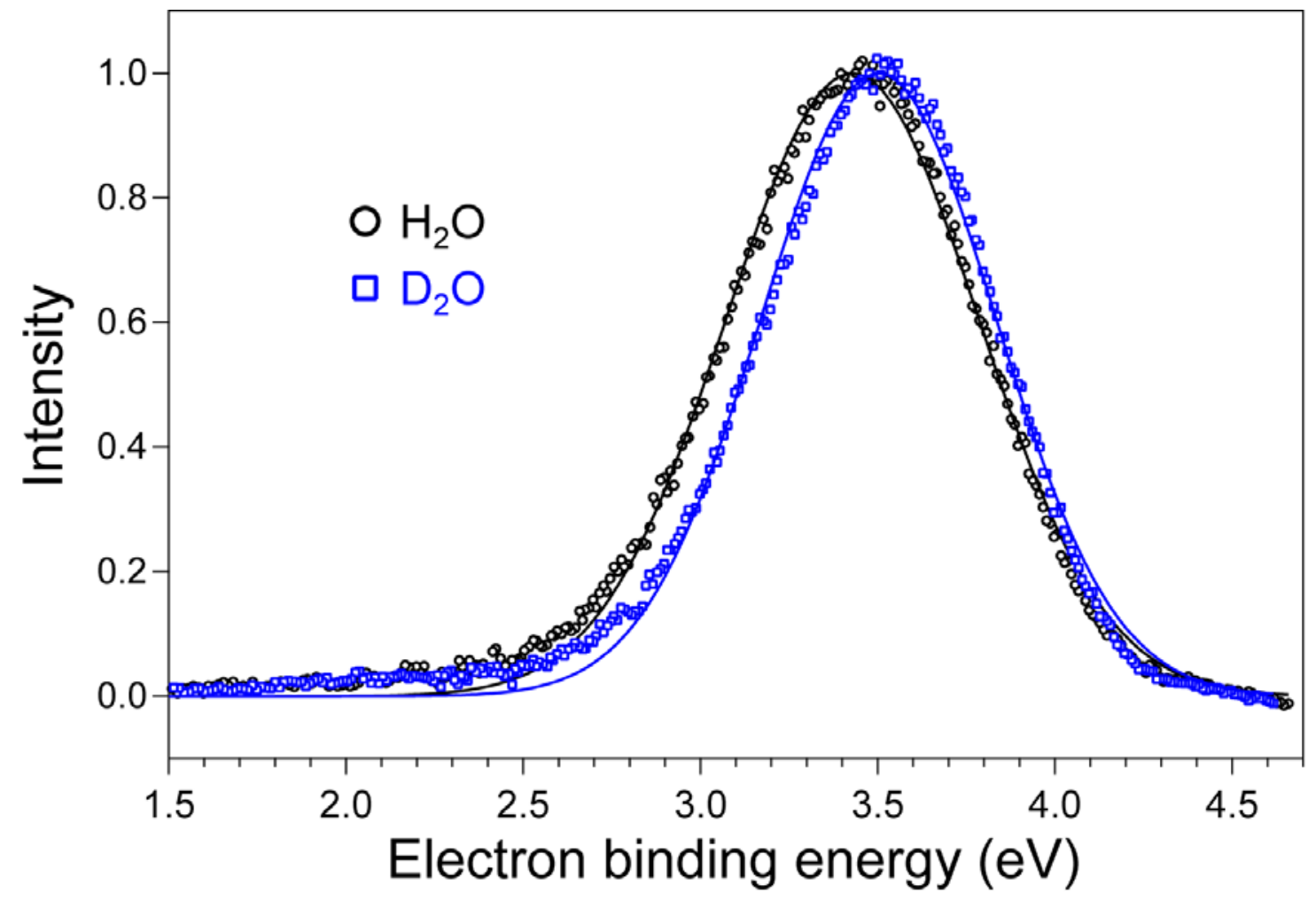

Figure 3. Horio et al

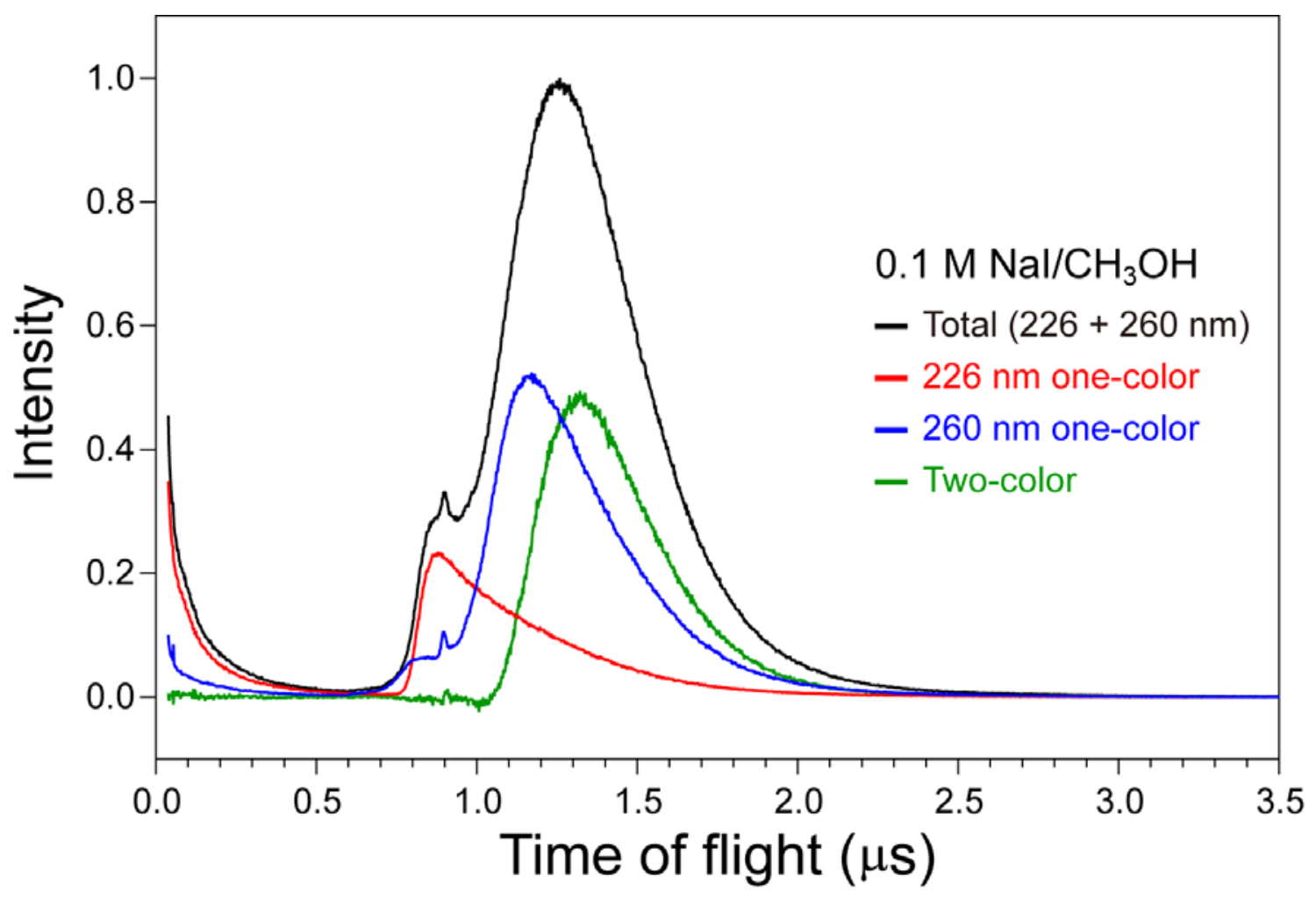

Figure 4. Horio et al 


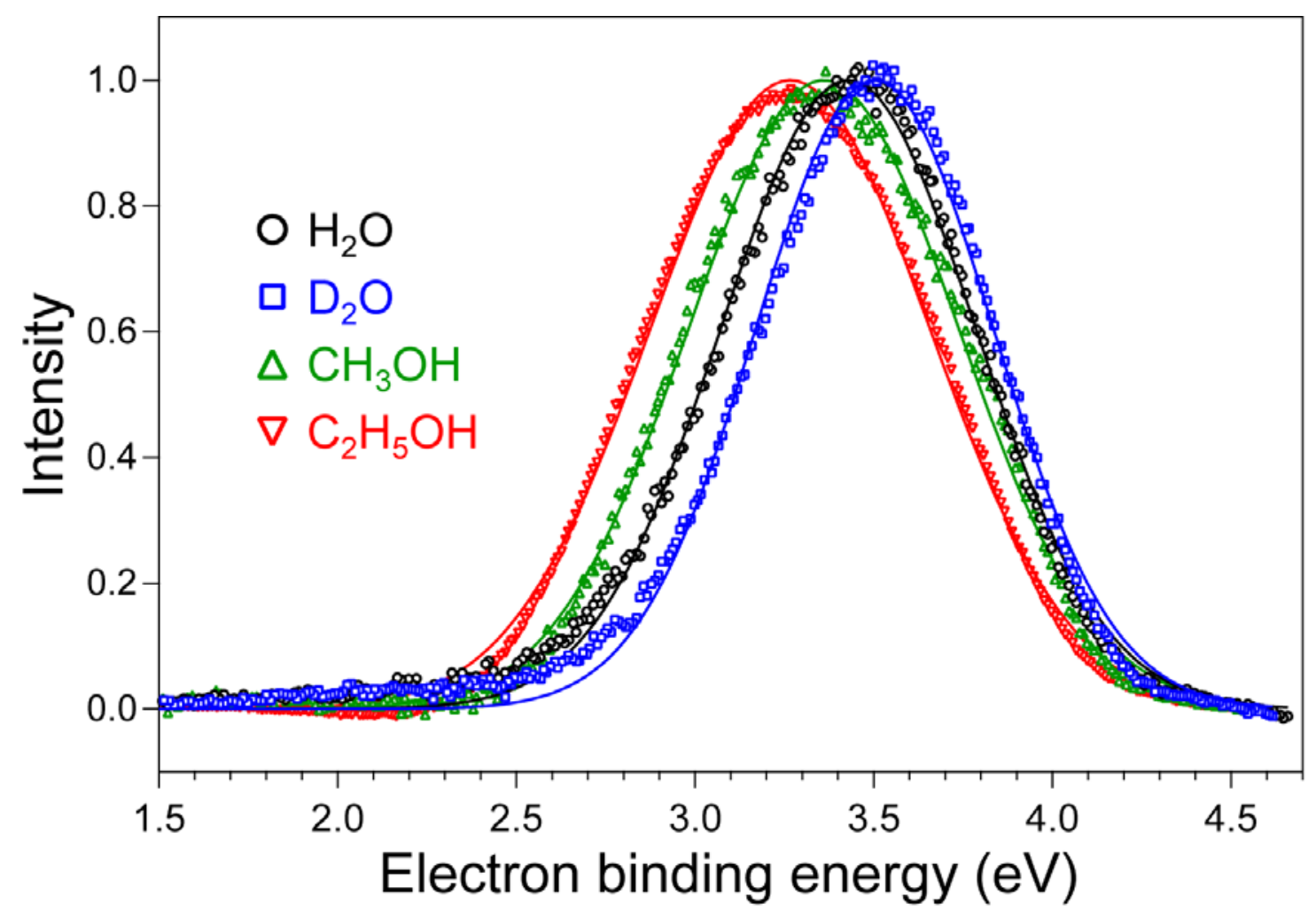

Figure 5. Horio et al

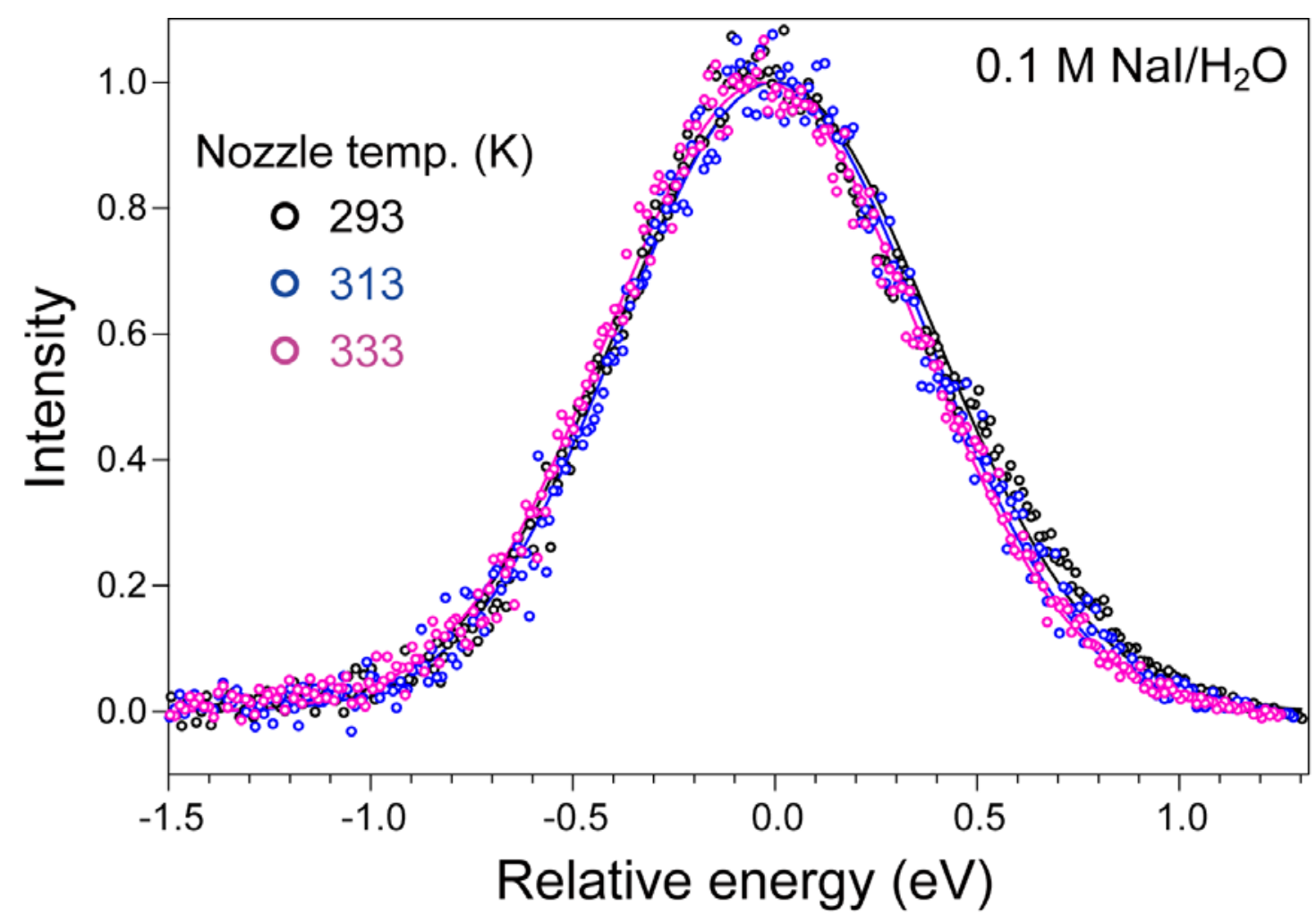

Figure 6. Horio et al 


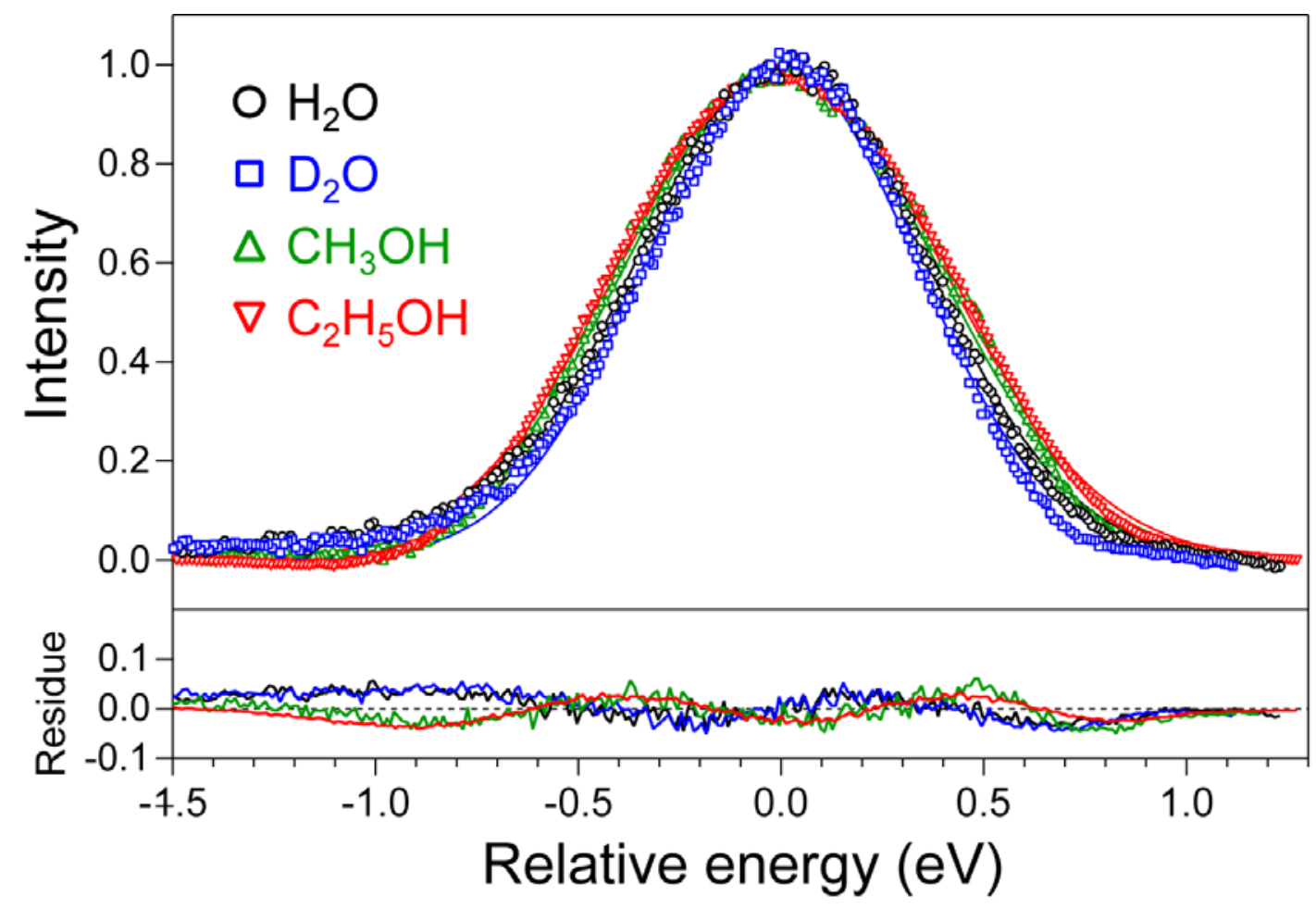

Figure 7. Horio et al 